\title{
Radiatively Driven Hypersonic Wind Tunnel
}

\author{
Richard B. Miles, ${ }^{*}$ Garry L. Brown, ${ }^{\dagger}$ Walter R. Lempert,,${ }^{\ddagger}$ Richard Yetter, ${ }^{\S}$ George J. Williams Jr.,, and Seymour M. Bogdonoff ${ }^{\|}$ \\ Princeton University, Princeton, New Jersey 08544 \\ Douglas Natelson** \\ Stanford University, Stanford, California 94305 \\ and \\ Jeffrey R. Guest ${ }^{\text {th }}$ \\ University of Michigan, Ann Arbor, Michigan 48109
}

\begin{abstract}
This paper outlines a new approach to the design of hypersonic wind tunnels. This new approach is motivated by fundamental limitations of conventional isentropic expansions that arise from the very high temperatures required to achieve the necessary enthalpy for hypersonic flow. These high temperatures lead to excessive throat degradation and contaminated air in the test section. The consequence is that the run times of conventional facilities must be short, and tests are conducted in "air" of unknown composition containing exceedingly high concentrations of radical and superequilibrium species such as $\mathrm{NO}$ as well as ablated plenum and throat material. The radiatively driven wind-tunnel approach takes advantage of the real gas properties of air to achieve high enthalpy at low temperature in the plenum, thus minimizing throat degradation and suppressing the formation of unwanted species in the plenum. Additional energy is radiatively added downstream of the throat in the expansion section to achieve the desired test conditions. The temperature of the air is kept low throughout the expansion, so that the formation of superequilibrium species and radicals is kept to a minimum. Radiative sources that couple to air include high-power lasers and microwave devices. A one-dimensional model including optical coupling is developed using, as an example, an $\mathrm{HF}$ laser coupled to the naturally occurring $\mathrm{CO}_{2}$ in air.
\end{abstract}

\section{Introduction}

$\mathbf{T}$ HE testing and evaluation of hypersonic airplane components and air-breathing propulsion systems are seriously limited by the availability of satisfactory ground test facilities. At Mach numbers higher than approximately Mach 10, tests under true simulated flight conditions, including correct atmospheric temperature and density, cannot be done with current or projected facilities. This limitation occurs because, in the conventional isentropic expansiontype wind-tunnel facility, temperatures in excess of $3000 \mathrm{~K}$ are required to achieve static temperatures on the order of $200 \mathrm{~K}$ or higher associated with true flight conditions. At these temperatures, the air cannot be contained for long periods of time due to materials limitations, and significant throat erosion occurs during wind-tunnel operation. In addition, radical and superequilibrium species such as NO are formed at high temperature and are frozen into the flowfield in the expansion, contaminating the flow. The solution to these problems has been to operate with very short run times to preserve the throat and to accept whatever contamination is present in the airflow. This short time operation severely limits the validity of component tests, and air contamination calls into question any propulsion measurements.

In this paper we examine a new approach to the design of a hypersonic facility. This approach leads to the possibility of long run times with properly constituted air (no significant contamination) at static

Received Aug. 10, 1994; revision received Jan. 20, 1995; accepted for publication Feb. 14, 1995. Copyright (C) 1995 by the American Institute of Aeronautics and Astronautics, Inc. All rights reserved.

*Professor, Department of Mechanical and Aerospace Engineering. Senior Member AIAA.

${ }^{t}$ Chairman, Department of Mechanical and Aerospace Engineering.

${ }^{*}$ Research Scientist, Department of Mechanical and Aerospace Engineering. Member AIAA.

${ }^{\S}$ Research Scientist, Department of Mechanical and Aerospace Engineering.

IGraduate Student, Department of Mechanical and Aerospace Engineering.

li Professor Emeritus, Department of Mechanical and Aerospace Engineering. Fellow AIAA.

${ }^{* *}$ Graduate Student, Physics Department.

${ }^{\dagger \dagger}$ Graduate Student, Applied Physics Department. temperatures and pressures characteristic of flight conditions. The basic concept is to maintain the air at moderate temperature but at ultrahigh pressure in the plenum, generating a cold, high-density flow at the throat. After an initial expansion to achieve supersonic flow, additional energy is radiatively coupled into the air as it expands through the nozzle, further accelerating it while simultaneously increasing the enthalpy and entropy. A final expansion to high Mach number brings the flow to the test conditions. With this approach, the air temperature is maintained low throughout the expansion so that thermally induced reactions and throat erosion are minimized.

An important aspect of this approach is that the ultrahigh pressure air in the plenum region has very high density and can no longer be treated as an ideal gas. The associated real gas effects cause the enthalpy to become pressure dependent, and so very high enthalpies can be achieved at modest temperatures. In addition, the speed of sound increases significantly, reaching values more typical of the speed of sound in liquid than in gas-phase air. As a consequence, the kinetic energy of the fluid passing through the throat at Mach 1 is much higher than would be possible for an ideal gas at a similar temperature.

A feature of this approach is that many parameters may be varied to achieve the desired flow. For example, the absorption of the air depends on its density, temperature, and pressure, as well as on the wavelength of the particular radiative source chosen. A change in the absorption will affect the length of the tunnel, which directly impacts the time the air spends at high temperature and, thus, the chemical dynamics, and so the choice of the wavelength can be optimized for the minimization of oxides of nitrogen. Other variables include the plenum pressure, the plenum temperature, the expansion profile, and the energy addition profile. More flexibility may be achieved by using multiple radiation sources, each optimized for a particular portion of the flow. A practical limit for the run time will be determined by the volume of high-pressure air that can be contained and the total stored energy of the radiative source. Run times in excess of $0.1 \mathrm{~s}$ are the goal of this approach.

\section{Technical Approach}

Overview

A convenient way of viewing the operation of such a facility is with the help of a Mollier diagram (Fig. 1). In this diagram, the 


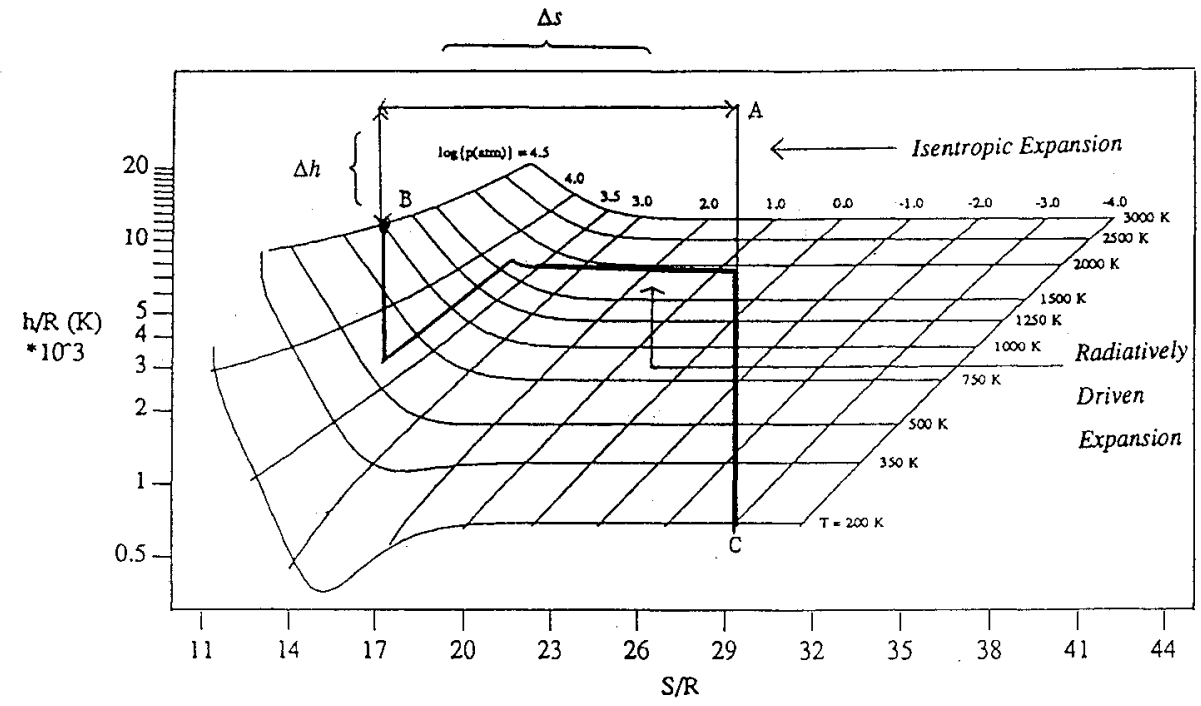

Fig. 1 Concept definition: thermodynamic comparison of wind-tunnel philosophies.

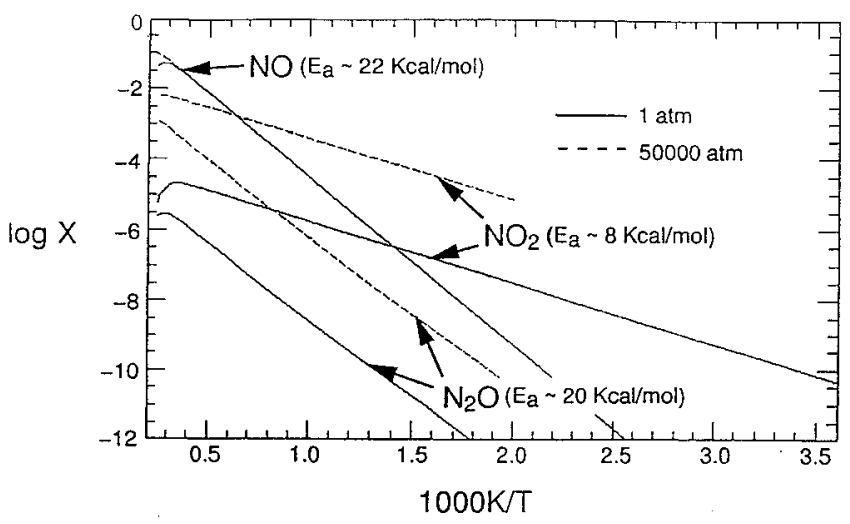

Fig. 2 Equilibrium mole fractions of $\mathrm{NO}, \mathrm{NO}_{2}$, and $\mathrm{N}_{2} \mathrm{O}$ in air at 1 and $50,000 \mathrm{~atm}$. For $1 \mathrm{~atm}$, the ideal gas equation of state was applied, and for $50 \mathrm{~atm}$, the Becker- Kistiakowsky-Wilson equation of state was assumed. ${ }^{1}$

enthalpy is plotted vs the entropy. For a conventional wind-tunnel facility, the expansion is assumed to be isentropic, and so the static temperature and pressure and the Mach number in the test chamber mandate a unique temperature and pressure for the gas in the plenum. For example, for a Mach 16 flow with a static temperature of $200 \mathrm{~K}$ and a static pressure of $10^{-3} \mathrm{~atm}$ (point $\mathrm{C}$ on the diagram), the plenum must be held at a temperature of $6300 \mathrm{~K}$ and at a pressure of $4000 \mathrm{~atm}$ (point A). Clearly, at this temperature, air is highly dissociated, and severe problems with containment and throat erosion occur. In contrast, the radiatively driven wind tunnel begins with a plenum pressure on the order of 30,000 atm but a plenum temperature on the order of $1000 \mathrm{~K}$. The point on the Mollier diagram (point B) associated with this initial condition corresponds to significantly lower entropy and lower enthalpy than that of the isentropic wind tunnel. That remaining enthalpy and entropy are then added downstream of the throat using the radiative source.

Chemistry is an important consideration in the design of the radiatively driven hypersonic facility concept. In the ideal case, the temperatures will be kept low enough so that the chemical kinetics can be ignored. In reality, however, there are two regimes in which chemical kinetics must be taken into account. The first is in the ultra-high-pressure plenum, where even at modest temperatures the formation of polyatomic molecules that have lower specific volume is favored. This effect is illustrated in Fig. 2. If equilibrium codes are extrapolated to $50,000 \mathrm{~atm}$ at $1000 \mathrm{~K}$, the predicted equilibrium mole fraction of $\mathrm{NO}_{2}$ increases by two orders of magnitude above its one atmosphere equilibrium concentration. The mole fraction of NO, on the other hand, is unaffected since it is a diatomic molecule and occupies the same molar volume as oxygen and nitrogen, which are both diatomic. At temperatures on the order of $1500 \mathrm{~K}$ or below, the rate of formation of those species is very slow, even at high pressure, and so a facility pulsed for times on the order of a second or less may never reach chemical equilibrium in the plenum.

The second regime of concern is in the region of heat addition where the temperatures increase substantially, and the formation rate of superequilibrium species is nonnegligible. Since the flow is moving at high speed (several kilometers/second), the residence time of the molecules in the high temperature regime is relatively short (milliseconds), and so the kinetic rate of formation of various molecular species becomes an important parameter. In particular, it is important to realize that the slow kinetic rate of formation of the nitric oxide molecule may suppress the nitric oxide concentrations for short times even though the temperature may be high.

One can generate an estimate of the temperature at which the additional enthalpy must be added downstream by recognizing that the total enthalpy (kinetic energy plus static enthalpy) and entropy are determined by the desired test conditions. Therefore, we can identify an enthalpy deficit $\Delta h$, which is the difference between the total enthalpy in the test chamber and the enthalpy in the plenum, and an entropy deficit $\Delta s$, which is the difference between the entropy of the gas in the test chamber and the entropy in the plenum. In the conventional isentropic facility, the enthalpy and entropy deficits are zero. Assuming an isentropic expansion, the total enthalpy in the test chamber is equal to the plenum enthalpy in the conventional facility, and so the enthalpy deficit is just the enthalpy difference between the plenum enthalpy of the conventional wind tunnel and the plenum enthalpy of the radiatively driven wind tunnel, as shown in Fig. 1. Similarly, the entropy deficit is the difference in plenum entropies as shown in the figure. When energy is added downstream of the throat, the ratio of these two deficits $(\Delta h / \Delta s)$ gives an "effective" temperature $T_{e}$. This is the effective temperature at which the additional enthalpy must be introduced downstream of the throat to achieve the proper entropy.

To minimize the formation of oxides of nitrogen and radicals in the nozzle section, it is desirable to keep this effective temperature as low as possible. This is accomplished by either decreasing $\Delta h$ (increasing the enthalpy in the plenum) or increasing $\Delta s$ (decreasing the entropy in the plenum). From Fig. 1 it is apparent that increasing the enthalpy in the plenum can be achieved by increasing the temperature or by increasing the pressure. Decreasing the entropy is accomplished by increasing the pressure or by decreasing the temperature. It is evident that high pressure is needed to keep the effective temperature low. Figure 3 shows the effective temperature as a function of plenum pressure for various plenum temperatures needed to achieve a Mach 16 flow at $200 \mathrm{~K}$ and $10^{-3}$ atm. 
Table 1 Compressibility factor constants

\begin{tabular}{llll}
\hline \hline$K(1)=6.243238 \times 10^{-2}$ & $K(12)=-7.271550 \times 10^{-4}$ & $K(23)=-8.451945 \times 10^{-2}$ \\
$K(2)=1.272148 \times 10^{-1}$ & $K(13)=-4.524547 \times 10^{-3}$ & $K(24)=-3.409313 \times 10^{-3}$ \\
$K(3)=-9.363323 \times 10^{-1}$ & $K(14)=1.304687 \times 10^{-3}$ & $K(25)=-1.951270 \times 10^{-3}$ \\
$K(4)=7.018441 \times 10^{-1}$ & $K(15)=-2.221651 \times 10^{-4}$ & $K(26)=4.938999 \times 10^{-5}$ \\
$K(5)=-3.516090 \times 10^{-1}$ & $K(16)=-1.981405 \times 10^{-3}$ & $K(27)=-4.932646 \times 10^{-5}$ \\
$K(6)=5.645003 \times 10^{-2}$ & $K(17)=5.975740 \times 10^{-5}$ & $K(28)=8.856666 \times 10^{-7}$ \\
$K(7)=2.995615 \times 10^{-2}$ & $K(18)=-3.641353 \times 10^{-6}$ & $K(29)=5.347880 \times 10^{-8}$ \\
$K(8)=-3.181744 \times 10^{-2}$ & $K(19)=8.413648 \times 10^{-6}$ & $K(30)=-5.934206 \times 10^{-8}$ \\
$K(9)=-1.682111 \times 10^{-2}$ & $K(20)=-9.828689 \times 10^{-9}$ & $K(31)=-9.068133 \times 10^{-9}$ \\
$K(10)=1.602041$ & $K(21)=-1.576831$ & $K(32)=1.618224 \times 10^{-9}$ \\
$K(11)=-1.099967 \times 10^{-3}$ & $K(22)=4.007290 \times 10^{-2}$ & $K(33)=-3.320448 \times 10^{-10}$
\end{tabular}

$$
\beta(\tau)=\sum_{i=1}^{6} K(i) \tau^{i-1} \quad \text { and } \quad \lambda=0.0588
$$

$\rho$ and $\tau$ are defined in terms of the critical values for the substance to be modeled:

$$
r=\frac{R T_{c} \rho}{P_{c}} \quad \text { and } \quad \tau=\frac{T_{c}}{T}
$$

where the critical parameters for air are approximated as

$T_{c}=132.5 \mathrm{~K} \quad P_{c}=3.77 \mathrm{MPa} \quad \rho_{c}=343.3 \mathrm{~kg} / \mathrm{m}^{3}$

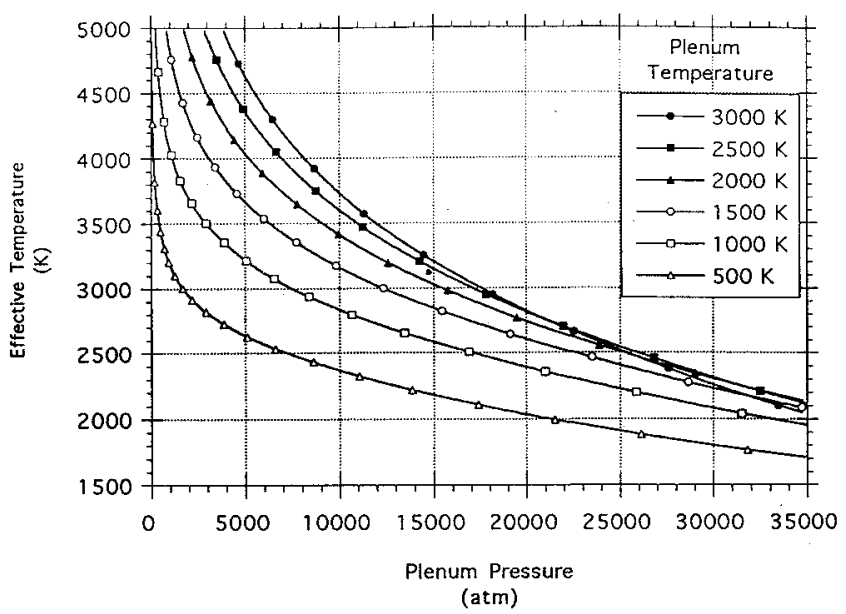

Fig. 3 Effective temperature vs plenum pressure for various plenum temperatures.

Balanced against this effective temperature are the maximum temperature reached in the expansion and the temperature in the plenum. In general, it is these temperatures and the time over which the flow is at these temperatures that determine the concentration of nitric oxide in the test region. Since the effective temperature is an average temperature at which the energy is added, if a significant amount of energy is added below the effective temperature, then an approximately equal amount must be added at a temperature higher than the effective temperature to achieve the proper test conditions. The maximum heating rate downstream of the throat is at constant pressure, since an increase in pressure will cause the flow to choke. Thus, to minimize the peak temperature, the flow should be heated isobarically until it reaches a temperature somewhat higher than the effective temperature, and then the remainder of the energy added. In the models presented here, we have chosen an initial expansion to Mach 2 before energy is added to avoid instabilities near Mach 1. Because of real gas effects, in the initial constant pressure heating region the Mach number does not drop significantly, even though the temperature increases by a factor of 3 or more.

\section{Flow Model}

A more accurate description of the operation of the wind tunnel can be developed using a one-dimensional real gas model. The governing equations are the continuity, momentum, and energy equations that are derived from a control volume analysis with heat input $\dot{q}$, expressed in joules per unit mass per unit length,

$$
\begin{gathered}
\rho u \frac{\mathrm{d} A}{\mathrm{~d} x}+\rho \frac{\mathrm{d} u}{\mathrm{~d} x} A+\frac{\mathrm{d} \rho}{\mathrm{d} x} u A=0 \\
\left(\frac{\partial P}{\partial \rho}\right)_{T} \frac{\mathrm{d} \rho}{\mathrm{d} x}+\left(\frac{\partial P}{\mathrm{~d} T}\right)_{\rho} \frac{\mathrm{d} T}{\mathrm{~d} x}=-\rho u \frac{\mathrm{d} u}{\mathrm{~d} x} \\
\left(\frac{\partial h}{\partial \rho}\right)_{T} \frac{\mathrm{d} \rho}{\mathrm{d} x}+\left(\frac{\partial h}{\partial T}\right)_{\rho} \frac{\mathrm{d} T}{\mathrm{~d} x}+u \frac{\mathrm{d} u}{\mathrm{~d} x}=\frac{\dot{q}}{\rho u A}
\end{gathered}
$$

where $\rho$ is the density, $h$ is the specific enthalpy, and $u$ is the velocity. The equation of state is ${ }^{2}$

$$
P=Z(\rho, T) \rho R T
$$

where the compressibility factor is given as

$$
\begin{aligned}
& Z=1+r \beta(\tau)+r^{2} \sum_{i=7}^{10} K(i) \tau^{i-7}+r^{3} \sum_{i=11}^{13} K(i) \tau^{i-11} \\
& +r^{4} K(14) \tau+r^{5}\left[K(15) \tau^{2}+K(16) \tau^{3}\right] \\
& \times r^{6} K(17) \tau^{2}+r^{7}\left[K(18) \tau^{2}+K(19) \tau^{3}\right] \\
& +r^{8} K(20) \tau^{3} \\
& +\left[\begin{array}{l}
r^{2}\left[K(21) \tau^{3}+K(22) \tau^{4}\right] \\
+r^{4}\left[K(23) \tau^{3}+K(24) \tau^{5}\right] \\
+r^{6}\left[K(25) \tau^{3}+K(26) \tau^{4}\right] \\
+r^{8}\left[K(27) \tau^{3}+K(28) \tau^{5}\right] \\
+r^{10}\left[K(29) \tau^{3}+K(30) \tau^{4}\right] \\
+r^{12}\left[K(31) \tau^{3}+K(32) \tau^{4}+K(33) \tau^{5}\right]
\end{array}\right] \bullet e^{-\lambda r^{2}}
\end{aligned}
$$

with $r$ being a dimensionless density based on the critical temperature $T_{c}$ and the critical pressure $P_{c}$,

$$
r=\rho \frac{R T_{c}}{P_{c}}
$$

and $\tau$ being a dimensionless temperature,

$$
\tau=\frac{T_{c}}{T}
$$

The constants $K(i)$ are listed in Table 1 . To find the other thermodynamic state variables, the ideal gas specific heat, $c_{v}^{0}(T)$, is used to 
find the low-density $\left(\rho=\rho_{\varepsilon}\right.$ ) (ideal gas) conditions at temperature $T$, and then the real gas equation is used to integrate at constant $T$ to the desired density. ${ }^{2}$ Therefore, for enthalpy and entropy,

$$
\frac{h-h^{0}}{R T_{c}}=\int_{r_{\varepsilon}}^{r} \frac{1}{r}\left(\frac{\partial Z}{\partial \tau}\right)_{r} \mathrm{~d} r+\frac{(z-1)}{\tau}
$$

and

$$
\frac{s-s^{0}}{R}=-\ln \left(\frac{\rho}{\rho_{\varepsilon}}\right)+\int_{r_{\varepsilon}}^{r} \frac{\tau}{r}\left(\frac{\partial Z}{\partial \tau}\right)_{r} \mathrm{~d} r-\int_{r_{\varepsilon}}^{r} \frac{1}{r}(Z-1) \mathrm{d} r
$$

where $r_{\varepsilon}=\rho_{\varepsilon}\left(R T_{c} / P_{c}\right)$ is effectively zero for the lower limits of the integrals, and $h^{0}$ contains the low-density specific enthalpies of oxygen and nitrogen and contains the low-density specific entropies of oxygen and nitrogen plus the entropy of mixing.

This model has been found to give an accurate reproduction of the Arnold Engineering and Development Center (AEDC) Mollier diagram that is derived from tabulated data, and it is consistent with similar real gas models published by the Soviet Bureau of Standards. ${ }^{3-5}$ This equation of state is solved using a finite difference solution to generate an aerodynamic model to describe the flow evolution with heating in the nozzle section. The model does not account for dissociation or high temperature and has been developed based on nitrogen data only up to 10,000 atm pressure at temperatures between 50 and $1200 \mathrm{~K}$. Extrapolations to higher pressure and temperature are smooth but have not been experimentally verified. The application of this model to air rather than nitrogen involves a single substance approximation for the critical pressure and temperature. To our knowledge there are no experimental data for air above $4550 \mathrm{~atm}^{6}$

This model was tested in several manners. First, with no energy addition and at constant area, calculated values of the density, temperature, and velocity were checked to confirm that these quantities remained constant throughout the flow. A second check was done modeling a diverging conical profile with initial conditions corresponding to the Mach number just greater than 1 and no energy addition. Calculated $\rho / \rho^{*}, T / T^{*}, P / P^{*}$, and $A / A^{*}$ profiles produced by the model were compared with tabulated solutions for an isentropic expansion. There was no perceptible deviation from the isentropic case. A third verification used the exactly solvable problem of energy addition into a flow with a constant cross-sectional area. The exact solution in this case is given by the expression

$$
\mathrm{d} h=\left[1-\frac{u^{2} \alpha}{c_{p}\left(1-M^{2}\right)}\right] \mathrm{d} s
$$

where $\alpha$ is the isobaric expansion coefficient,

$$
\alpha=\frac{1}{v}\left(\frac{\partial v}{\partial T}\right)_{p}
$$

and $v$ is the specific volume $(1 / \rho)$. Both $\alpha$ and the specific heat $c_{p}$ were calculated numerically, and the ratio $(\mathrm{d} h / \mathrm{d} x) /(\mathrm{d} s / \mathrm{d} x)$ was normalized by Eq. (10) and checked to be sure that the value remained unity as the flow evolved down the constant area duct. The result was accurate to one part in $10^{6}$, independent of $\rho, T, v$, and $\dot{q}$ for appropriately small step sizes. The computation uses as an independent variable either the area profile $A(x)$, the pressure profile $p(x)$, or the temperature profile $T(x)$. The energy deposition rate $\dot{q}(x)$ can either be specified, or it can be derived from the absorption characteristics of the air, which are modeled as a function of temperature and pressure.

\section{Energy Addition}

The performance of the wind tunnel depends on both the plenum conditions and on the mechanism for energy addition. Using current technology, the maximum plenum pressure achievable is estimated to be between 20,000 and 30,000 atm. Energy must be added to the moving flow, and so radiative coupling is an obvious approach. This coupling must be accomplished via absorptive energy transitions that occur in air. The very high pressure of the air just downstream of the throat will lead to significant pressure dependence
Conventional Hypersonic Tunnel

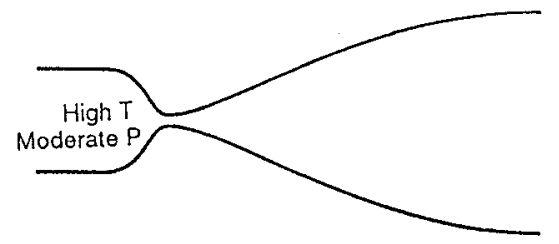

Radiation-driven Hypersonic Tunnel
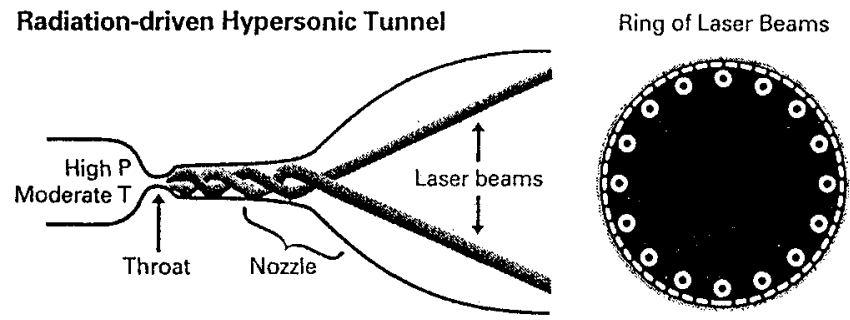

Fig. 4 Schematic comparison of wind-tunnel philosophies.

(both pressure narrowing and pressure broadening) of absorption features, and at these high pressures, pressure-induced absorption phenomena are often dominant. Transitions occurring in the infrared region are accessible to various high-power lasers, and those in the microwave region can be pumped by high-power gyrotron devices. Of particular interest are pressure-induced transitions in oxygen in the near infrared that overlap diode lasers and diode-pumped, solidstate lasers; $\mathrm{CO}_{2}$ transitions that overlap hydrogen fluoride (HF) and reactor pumped xenon laser lines; $\mathrm{H}_{2} \mathrm{O}$ transitions that overlap the chemical oxygen iodine laser (COIL); and the oxygen microwave transition at $60 \mathrm{GHz}$. Other transitions to be explored include $\mathrm{H}_{2} \mathrm{O}$ and $\mathrm{CO}_{2}$ lines that overlap $\mathrm{CO}_{2}$ and $\mathrm{CO}$ lasers and pressure-induced oxygen and nitrogen transitions throughout the infrared. Ultraviolet transitions, particularly those in oxygen, might also be explored with high-power light sources and, possibly, with ultraviolet lasers.

As a test case, the possibility of adding energy optically with high-power HF lasers was studied. Although the calculation is one dimensional, a possible tunnel geometry is sketched in Fig. 4. The lasers are assumed to enter the tunnel from downstream, passing through the test section and into the nozzle. They are arranged in a ring so as not to interfere with the model. Since most of the absorption occurs where the air is at high pressure, this configuration has the potential of efficiently coupling energy into the flow just downstream of the throat. To compute the flow, an initial guess of the energy deposition parameter $\dot{q}$ through the nozzle is given, and the model is integrated in the forward direction to solve for the air density and temperature through the expansion, and then in the backward direction (from the test section back toward the throat) to give a second iteration on the energy addition profile based on the energy absorbed by the air at the computed temperature and density. This procedure is carried out repeatedly until convergence is achieved. Following convergence, the energy addition rate profile is consistent with the absorptivity profile of the expanding airstream.

Although the thermodynamic properties of the gas appear to be good approximations to the actual thermodynamic state of air over these ranges of pressure and temperature, the absorption constant is only a crude estimate. In the example worked here, the high-power HF lasers are assumed to be operating on a single transition near $2.76 \mu\left(3623 \mathrm{~cm}^{-1}\right)$. Absorption occurs through the small amount of $\mathrm{CO}_{2}$ that is present in air and reflects the upper atmospheric mole fraction ratio of 330 parts/million. The absorption is directly into a bending/asymmetric stretch (021) combination band, and absorption data are taken from the HITRAN database, extrapolated to high pressure using conventional pressure broadening. ${ }^{7}$ Since pressure narrowing effects are ignored, the values that are used only serve to illustrate this approach to energy addition and cannot be interpreted quantitatively. Figure 5 shows the absorption profile in the vicinity of the HF line at a pressure of $1000 \mathrm{~atm}$. To accommodate our uncertainty in the absorption line shape and magnitude, a pressuredependent absorption constant equivalent to one-half of that shown on the figure for the $P_{2}(4)$ line was used for the calculation. 
Table 2 Computed gases

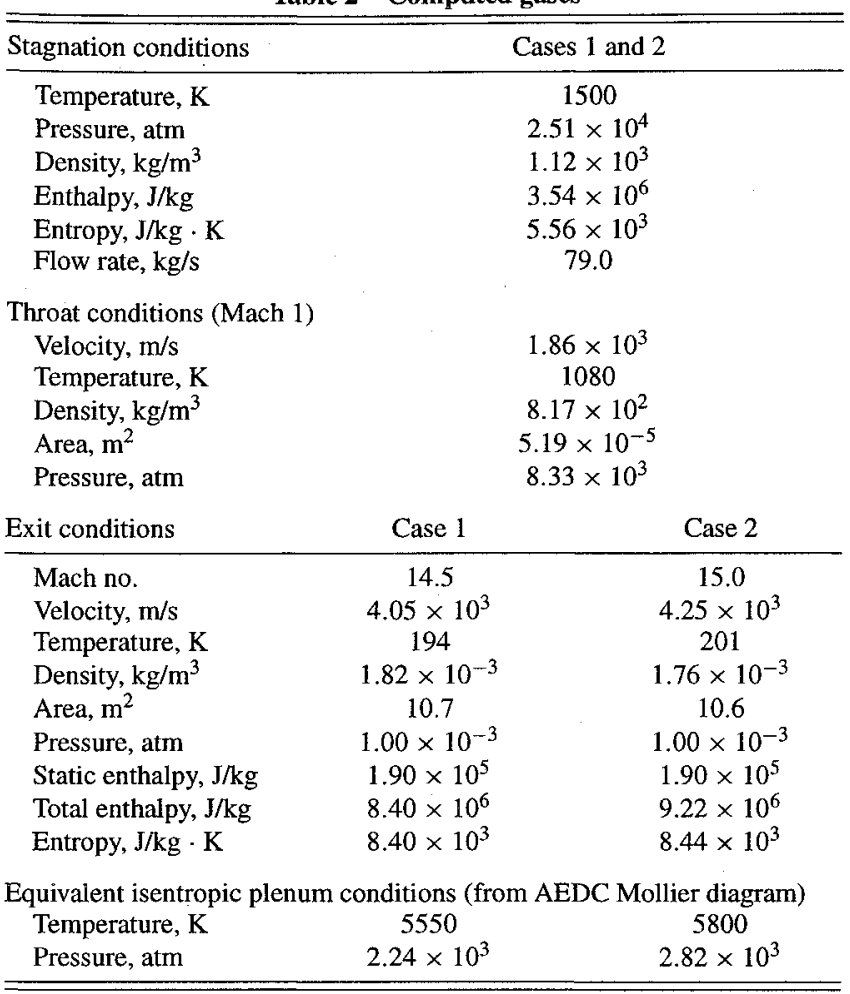

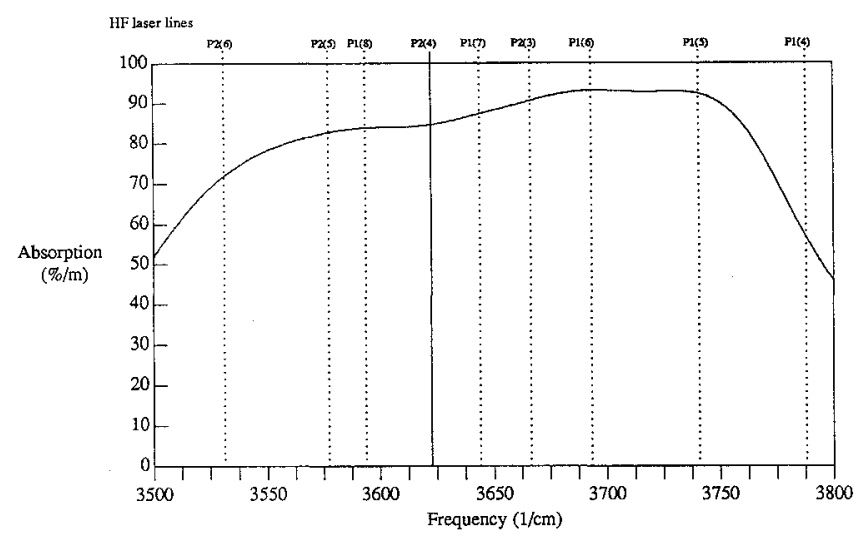

Fig. $5 \mathrm{CO}_{2}$ absorption (percent per meter) for air at 1000 atm and $1000 \mathrm{~K}$. Vertical lines correspond to HF laser lines.

Two examples are given to demonstrate the effect of different expansions. Both cases begin with the same plenum pressure, 25,120 atm, and temperature, $1500 \mathrm{~K}$, and are expanded to roughly $200 \mathrm{~K}$ and $10^{-3} \mathrm{~atm}$. Mach numbers of 14.5 and 15.0 are achieved by cases 1 and 2, respectively. The stagnation conditions, throat conditions, and conditions in the test section for the computed cases are shown in Table 2. The constraint applied for the calculation was initial energy addition at constant pressure, followed by isothermal energy addition, and a final expansion to the test cell that has approximately a 3-m diameter. In the first case, the flow is heated at constant pressure to $2000 \mathrm{~K}$ and then heated at constant temperature. Since the code is one dimensional, the length and shape of the final expansion are arbitrary. The shape of the final expansion is chosen here to produce a smooth pressure drop to $10^{-3}$ atm pressure, and the length of the tunnel is chosen to be $40 \mathrm{~m}$. In the second case, the flow is heated at constant pressure up to $2500 \mathrm{~K}$ and then heated at constant temperature until the proper entropy is reached. The initial conditions for the energy addition portion of the computation begin at Mach 2 in both cases, and so the flow passes through the throat and expands to Mach 2 before heat addition begins. In both cases, the optical energy is fully absorbed downstream of the Mach 2 position by allowing sufficient separation between the nozzle location and the energy addition regime, and so there is no truncation error associated with these initial conditions.

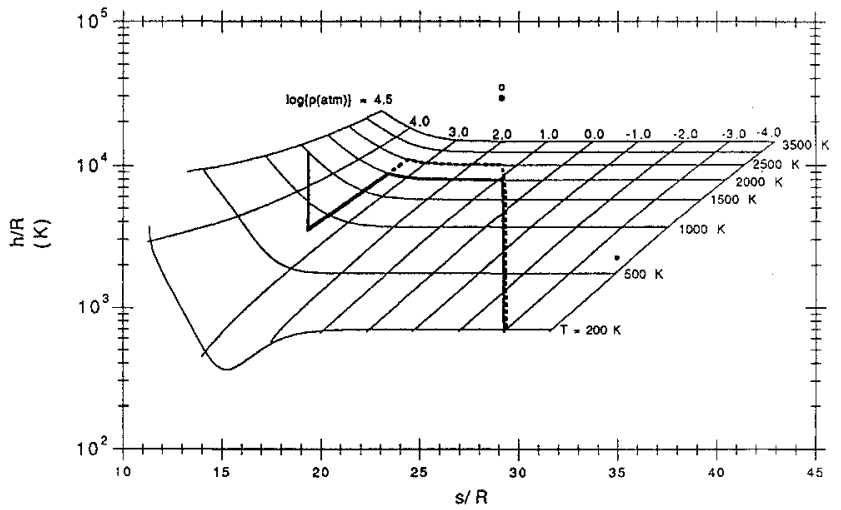

Fig. 6 Mollier diagram for cases $1(2000 \mathrm{~K} \rightarrow$ solid line, solid dot) and 2 ( $2500 \mathrm{~K}$-dotted line, open dot).

Figure 6 shows the trajectories of cases 1 and 2 on the Mollier diagram. The points at the top of the diagram show the initial conditions for an isentropic expansion to achieve the same test conditions. These numbers are taken from the AEDC Mollier diagram since our model is not designed for exceedingly high temperature.

Figures $7 \mathrm{a}-7 \mathrm{f}$ show the pressure, temperature, tunnel radius, Mach number, flow velocity, and heating profile as a function of the axial distance downstream for these two cases. It is particularly striking to note the large change in tunnel radius as a function of axial distance. Figures $8 \mathrm{a}$ and $8 \mathrm{~b}$ show an expanded view of the radius and the heating profile over the first few meters. Because of the very high density of the fluid at the throat, the radius at that location is less than $5 \mathrm{~mm}$. Apparent discontinuities in these curves are due to discontinuous changes in the modeled expansion profiles or energy addition constraints. As the flow proceeds downstream, energy addition occurs primarily over the first few meters, after which the secondary expansion increases the radius to on the order of $1.7 \mathrm{~m}$. The fluid flow characteristics at the throat are shown in Table 2. Note that the velocity at the throat is $1,860 \mathrm{~m} / \mathrm{s}$. This sonic speed would correspond to an "ideal gas" at a temperature in excess of $8000 \mathrm{~K}$, whereas the flow temperature is actually $1080 \mathrm{~K}$.

In case 2 , significantly more energy is coupled into the flow since it is heated to $2500 \mathrm{~K}$ rather than $2000 \mathrm{~K}$ before the isothermal energy addition begins. This is reflected both in the test conditions and in the effective temperatures. In case 1 , the effective temperature is $1740 \mathrm{~K}$, the flow velocity in the test section is $4.05 \mathrm{~km} / \mathrm{s}$, and the static temperature is $194 \mathrm{~K}$. For case 2 , the effective temperature is $1970 \mathrm{~K}$, the flow velocity is $4.25 \mathrm{~km} / \mathrm{s}$, and the temperature in the test section is $201 \mathrm{~K}$.

\section{Flow Chemistry}

An important design goal for this wind-tunnel facility is to match the molecular concentrations that are found at the flight altitudes simulated. Particular difficulty arises with regard to the molecular concentration of nitric oxide, since that species is formed in abundance at high temperature and tends to freeze into the flow. Nitric oxide concentrations in the upper atmosphere range from on the order of 50 parts/billion up to $80 \mathrm{~km}(260,000 \mathrm{ft})$ and then increase to a maximum of approximately 200 parts/million at 110 $\mathrm{km}(360,000 \mathrm{ft}){ }^{8}$ These mole fractions correspond to equilibrium temperatures ranging from approximately 600 to $1200 \mathrm{~K}$, according to the equilibrium diagram shown in Fig. 2. An important feature of this facility is that, even though the air must reach temperatures higher than these numbers, the time at which it remains at those temperatures is short, and therefore the nitric oxide concentrations are significantly suppressed below their equilibrium levels. This occurs both in the plenum where, even though the pressure is very high, the temperature is low, and so the kinetic formation rate of nitric oxide is on the order of seconds, and in the expansion, where the temperatures are higher but the times for formation are only on the order of milliseconds.

To compute the NO concentrations, we have applied an elementary chemical kinetic model based on 23 principal air reactions and the real gas Becker-Kistiakowsky-Wilson equation of state. These 


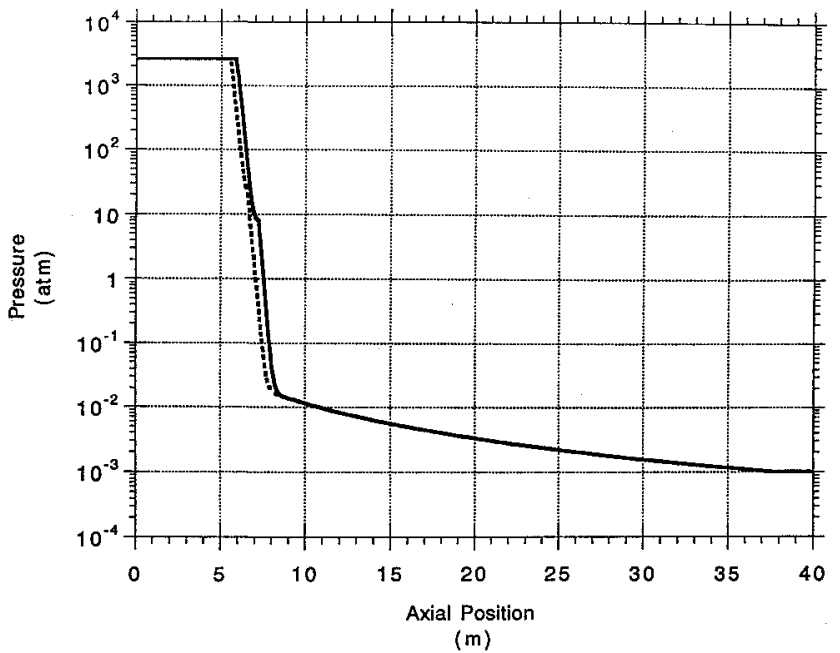

a) Pressure

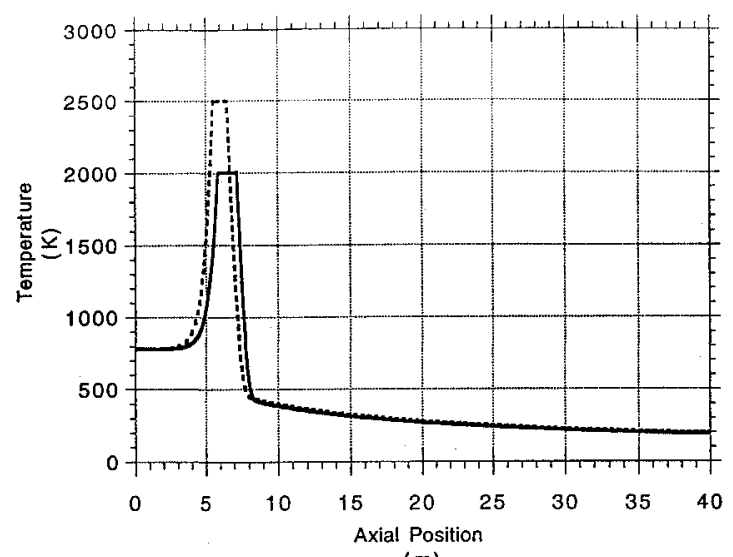

b) Temperature

(m)

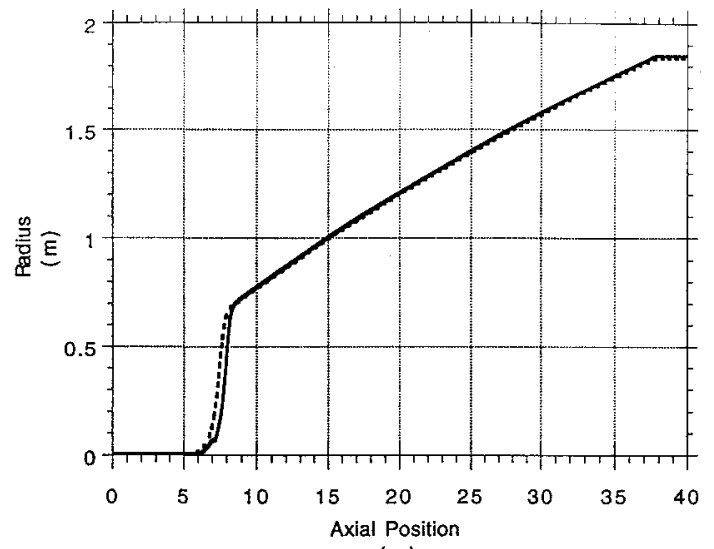

c) Radius

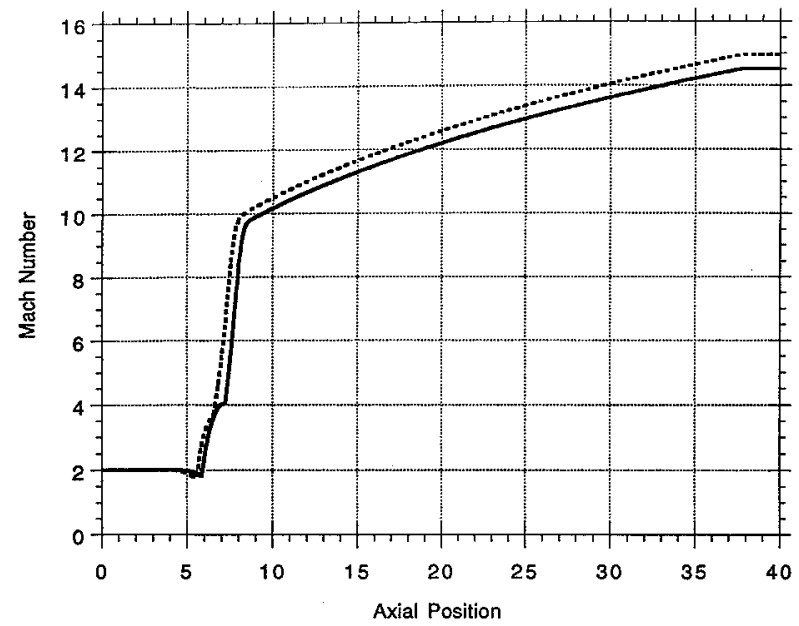

(m)

d) Mach number

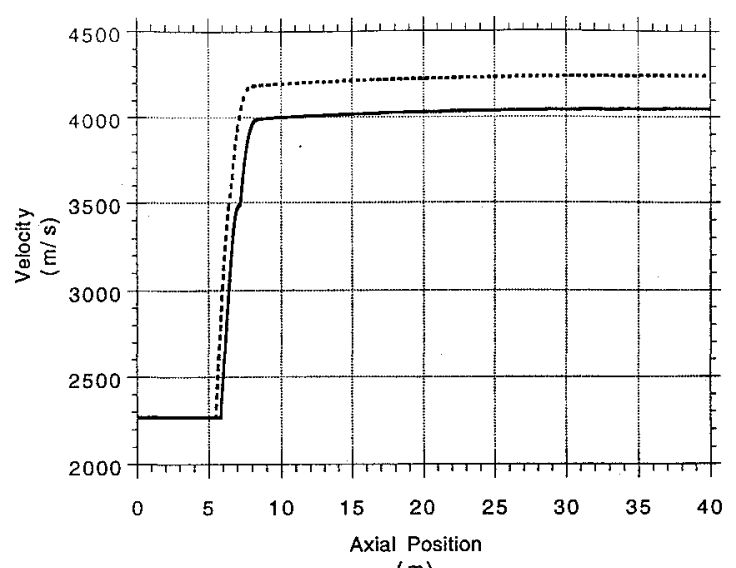

$(\mathrm{m})$

e) Velocity

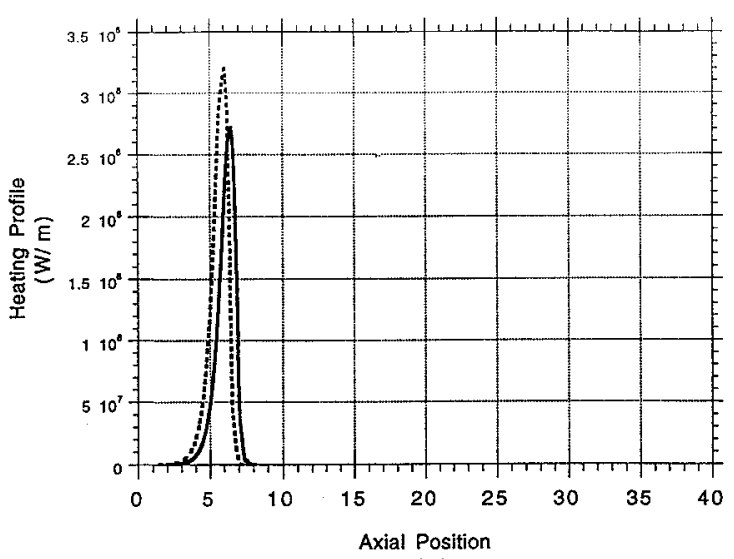

(m)

f) Heating profile

Fig. 7 Tunnel parameters for cases 1 (2000 K-solid line) and 2 (2500 K-dotted line).

reactions and the pressure-dependent rates are given in Table 3. Nitric oxide, oxygen radical, and $\mathrm{NO}_{2}$ concentrations for the two cases discussed earlier are shown in Figs. 9 and 10. It is important to note that the equilibrium molar concentration for nitric oxide at 2000 $\mathrm{K}$ is approximately $10^{-2}(1 \%)$. Here we see that in case 1 the formation of nitric oxide has been dramatically suppressed by the slow chemical formation rates. In case 2 , however, the flow is maintained at $2500 \mathrm{~K}$ for a relatively long period of time, and the nitric oxide concentration comes close to its equilibrium value. These curves suggest that various different scenarios for energy addition will have a significant impact on the level of nitric oxide formed. The concentrations of other nonequilibrium species are lower than NO but cannot be neglected.
In this model, the time delay between the excitation of the $\mathrm{CO}_{2}$ molecule and the deposition of energy into the kinetic motion of the flow is modeled assuming a single vibrational temperature. Although the exact mechanisms for this energy transfer remain to be explored, it is expected that the $\mathrm{CO}_{2}$ molecule will rapidly transfer its energy to nitrogen by exciting the vibrational mode of the nitrogen molecule. At low pressure, vibrationally excited nitrogen has a very long relaxation time. At high pressures, however, nitrogen rapidly transfers its excitation to oxygen, which is rapidly thermalized. Accurate modeling of both the collisional energy transfer from $\mathrm{CO}_{2}$ to nitrogen and the nitrogen relaxation mechanism will be important since many photons must be transferred to each $\mathrm{CO}_{2}$ molecule to achieve the required heating rates. The 
Table 3 High-temperature air reaction mechanism ${ }^{\mathrm{a}}$

\begin{tabular}{|c|c|c|c|c|c|c|c|c|c|}
\hline & Reaction $^{b}$ & $A$ & $n$ & $E$ & $A_{0}$ & $n_{0}$ & $E_{0}$ & $a$ & $b$ \\
\hline 1 & $\mathrm{O}_{2}(+\mathrm{M})=2 \mathrm{O}(+\mathrm{M})$ & $0.15 \times 10^{12}$ & 0.0 & $118,000.0$ & $0.98 \times 10^{25}$ & -2.5 & $118,000.0$ & - & - \\
\hline 2 & $\mathrm{O}+\mathrm{O}_{2}(+\mathrm{M})=\mathrm{O}_{3}(+\mathrm{M})$ & $0.17 \times 10^{13}$ & 0.0 & -0.0 & $0.18 \times 10^{22}$ & -2.8 & -0.0 & 0.65 & - \\
\hline 3 & $\mathrm{O}+\mathrm{O}_{3}=2 \mathrm{O}_{2}$ & $0.12 \times 10^{14}$ & 0.0 & -4.5 & - & - & - & - & $\longrightarrow$ \\
\hline 4 & $\mathrm{CO}+\mathrm{O}+\mathrm{M}=\mathrm{CO}_{2}+\mathrm{M}$ & $\longrightarrow$ & - & - & $0.25 \times 10^{14}$ & 0.0 & $-4,541.0$ & - & 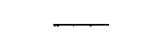 \\
\hline 5 & $\mathrm{CO}+\mathrm{O}_{2}=\mathrm{CO}_{2}+\mathrm{O}$ & $0.25 \times 10^{13}$ & 0.0 & $47,690.0$ & - & - & - & - & $\longrightarrow$ \\
\hline 6 & $\mathrm{NO}(+\mathrm{M})=\mathrm{N}+\mathrm{O}(+\mathrm{M})$ & $0.15 \times 10^{12}$ & 0.0 & $148,000.0$ & $0.15 \times 10^{16}$ & 0.0 & $148,400.0$ & - & - \\
\hline 7 & $\mathrm{NO}+\mathrm{O}(+\mathrm{M})=\mathrm{NO}_{2}(+\mathrm{M})$ & $0.13 \times 10^{16}$ & -0.75 & 0.0 & $0.47 \times 10^{25}$ & -2.87 & $1,550.0$ & 0.95 & $1.0 \times 10^{-4}$ \\
\hline 8 & $\mathrm{NO}+\mathrm{O}=\mathrm{O}_{2}+\mathrm{N}$ & $0.18 \times 10^{10}$ & 1.0 & $38,760.0$ & $\ldots$ & - & - & — & $\ldots$ \\
\hline 9 & $2 \mathrm{NO}=\mathrm{N}_{2}+\mathrm{O}_{2}$ & $0.13 \times 10^{15}$ & 0.0 & $75,630.0$ & & & & & \\
\hline 10 & $\mathrm{NO}_{2}+\mathrm{O}=\mathrm{O}_{2}+\mathrm{NO}$ & $0.39 \times 10^{13}$ & 0.0 & -238.4 & - & $\longrightarrow$ & 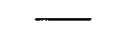 & - & - \\
\hline 11 & $\mathrm{NO}_{2}+\mathrm{O}(+\mathrm{M})=\mathrm{NO}_{3}(+\mathrm{M})$ & $0.13 \times 10^{14}$ & 0.0 & 0.0 & $0.15 \times 10^{29}$ & -4.08 & $1,467.0$ & 0.79 & $2.5 \times 10^{-4}$ \\
\hline 12 & $\mathrm{NO}_{2}+\mathrm{NO}=\mathrm{N}_{2} \mathrm{O}+\mathrm{O}_{2}$ & $0.10 \times 10^{13}$ & 0.0 & $60,000.0$ & 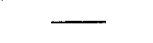 & & & & \\
\hline 13 & $2 \mathrm{NO}_{2}=\mathrm{NO}_{3}+\mathrm{NO}$ & $0.96 \times 10^{10}$ & 0.73 & $20,920.0$ & & & & & 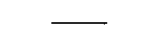 \\
\hline 14 & $2 \mathrm{NO}_{2}=2 \mathrm{NO}+\mathrm{O}_{2}$ & $0.16 \times 10^{13}$ & 0.0 & $26,120.0$ & - & - & - & - & - \\
\hline 15 & $\mathrm{~N}_{2} \mathrm{O}(+\mathrm{M})=\mathrm{N}_{2}+\mathrm{O}(+\mathrm{M})$ & $0.13 \times 10^{12}$ & 0.0 & $59,610.0$ & $0.72 \times 10^{18}$ & -0.73 & $62,790.0$ & 1.17 & $1.25 \times 10^{-4}$ \\
\hline 16 & $\mathrm{~N}_{2} \mathrm{O}+\mathrm{O}=\mathrm{N}_{2}+\mathrm{O}_{2}$ & $0.10 \times 10^{15}$ & 0.0 & $28,020.0$ & & $\square$ & $\longrightarrow$ & $\longrightarrow$ & 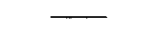 \\
\hline 17 & $\mathrm{~N}_{2} \mathrm{O}+\mathrm{O}=2 \mathrm{NO}$ & $0.66 \times 10^{14}$ & 0.0 & $26,630.0$ & & & & & - \\
\hline 18 & $\mathrm{~N}_{2} \mathrm{O}+\mathrm{NO}=\mathrm{N}_{2}+\mathrm{NO}_{2}$ & $0.10 \times 10^{15}$ & 0.0 & $49,675.0$ & & & & & \\
\hline 19 & $\mathrm{~N}+\mathrm{NO}=\mathrm{N}_{2}+\mathrm{O}$ & $0.33 \times 10^{13}$ & 0.3 & 0.0 & - & $\underline{.}$ & $\longrightarrow$ & $\longrightarrow$ & - \\
\hline 20 & $\mathrm{~N}+\mathrm{N}_{2} \mathrm{O}=\mathrm{N}_{2}+\mathrm{NO}$ & $0.10 \times 10^{14}$ & 0.0 & $19,870.0$ & & & & & \\
\hline 21 & $\mathrm{~N}+\mathrm{NO}_{2}=2 \mathrm{NO}$ & $0.40 \times 10^{13}$ & 0.0 & 0.0 & & & + & - & \\
\hline 22 & $\mathrm{~N}+\mathrm{NO}_{2}=\mathrm{N}_{2} \mathrm{O}+\mathrm{O}$ & $0.50 \times 10^{13}$ & 0.0 & 0.0 & - & $\overline{-}$ & $-\cdots$ & $\longrightarrow$ & - \\
\hline 23 & $\mathrm{~N}+\mathrm{NO}_{2}=\mathrm{N}_{2}+\mathrm{O}_{2}$ & $0.10 \times 10^{13}$ & 0.0 & 0.0 & - & . & & & \\
\hline
\end{tabular}

${ }^{\mathrm{a}}$ Units are $\mathrm{cm}^{3}$-mole-s-cal, $k=A T^{n} \exp \left(-E_{a} / R T\right)$.

${ }^{b}$ For pressure-dependent reactions with the total concentration, $M$ listed in parentheses, fall-off behavior is included as follows:

$$
\begin{aligned}
& k=\left\{\frac{k_{0} k_{\infty}}{k_{0}+\left(k_{\infty} /[M]\right)}\right\} \times F \\
& \log F=\frac{\log F_{c}}{\left(1+\left[\log \left(k_{0} \times[M] / k_{\infty}\right)\right]^{2}\right\}} \\
& k_{0}=A_{0} T^{n_{0}} \exp \left(-E_{0} / R T\right) \\
& k_{\infty}=A T^{n} \exp (-E / R T) \\
& F_{c}=a-b T
\end{aligned}
$$

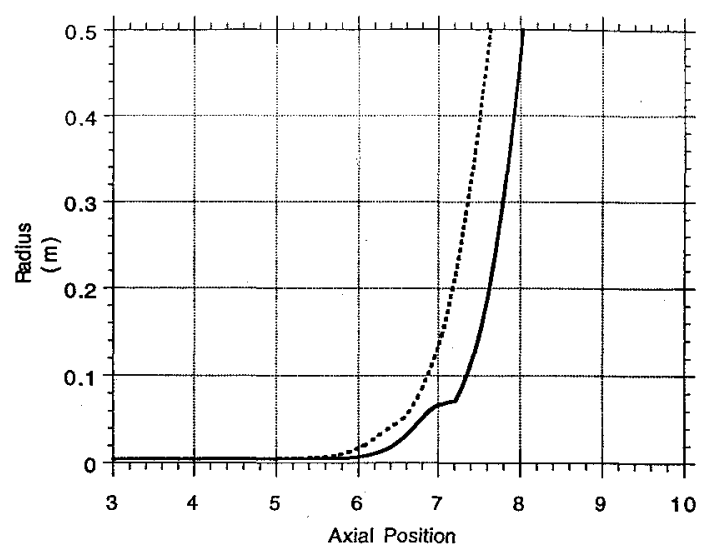

(m)

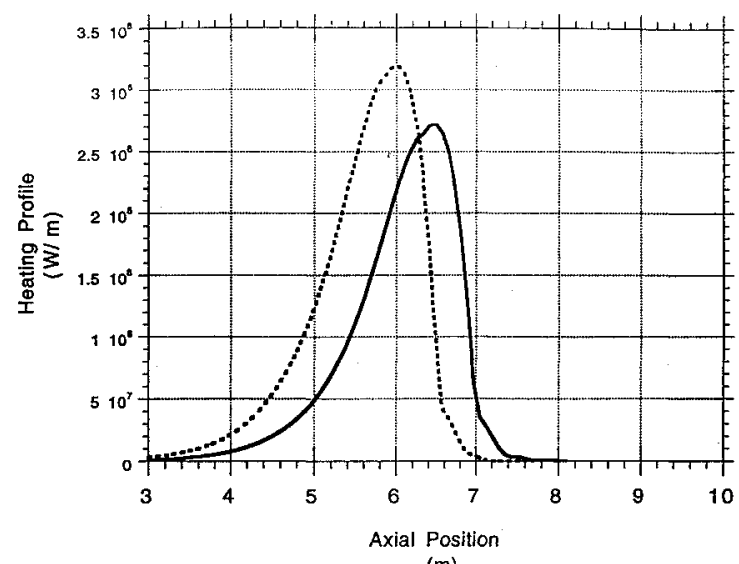

(m)

a) Radius

\section{b) Heating profile}

Fig. 8 Expanded scale wind-tunnel parameters for cases 1 (2000 K-solid line) and 2 (2500 K-dotted line).

scale of this energy deposition problem can be better appreciated by realizing that the enthalpy deficits in cases 1 and 2 are 4.9 and 5.7 million $\mathrm{J} / \mathrm{kg}$, respectively. This corresponds to approximately 10,000 photons per $\mathrm{CO}_{2}$ molecule, all of which must be absorbed in a few milliseconds, and so collisional transfer rates shorter than $0.1 \mu \mathrm{s}$ are required. In a large-scale facility such as this, a flow rate on the order of $80 \mathrm{~kg} / \mathrm{s}$ will be necessary. Thus, a total energy deposition rate of $400-450 \mathrm{MW}$ must be achieved over the run time of the tunnel. This involves a significant scaling of candidate laser sources and the use of multiple lasers. Tunnel operation for $0.1 \mathrm{~s}$ will require a total energy of $50 \mathrm{MJ}$ to be deposited into the flow. By comparison, this is approximately the amount of energy stored in
$20 \mathrm{gal}$ of gasoline and is well within the current total energy capability of existing high-power hydrogen fluoride/deuterium fluoride (HF/DF) lasers.

\section{Alternate Approaches}

The use of an $\mathrm{HF}$ laser to achieve energy addition via a $\mathrm{CO}_{2}$ transition has the advantage that very high-power HF and DF lasers have already been developed through Department of Defense support. For example, the MIRACL laser currently is capable of operating at power levels in excess of $1 \times 10^{6} \mathrm{~W}$. Further scaling of that laser to power levels on the order of $300 \times 10^{6} \mathrm{~W}$ will constitute 


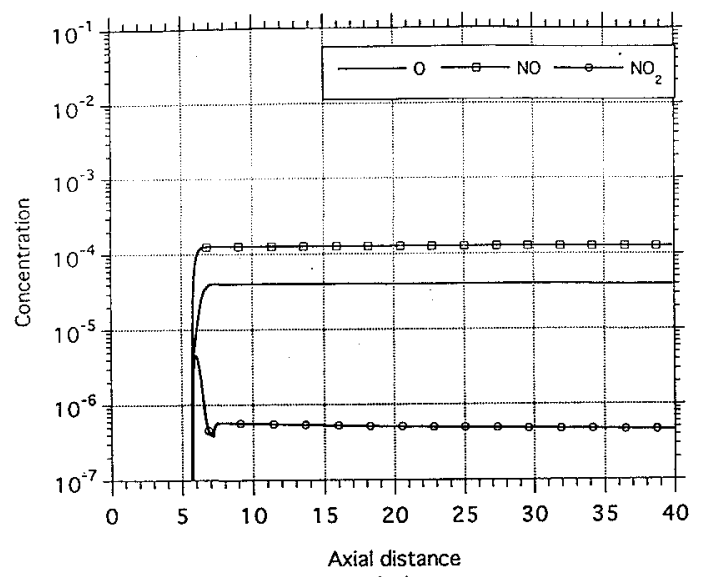

(m)

Fig. 9 Nonequilibrium concentrations for case 1.

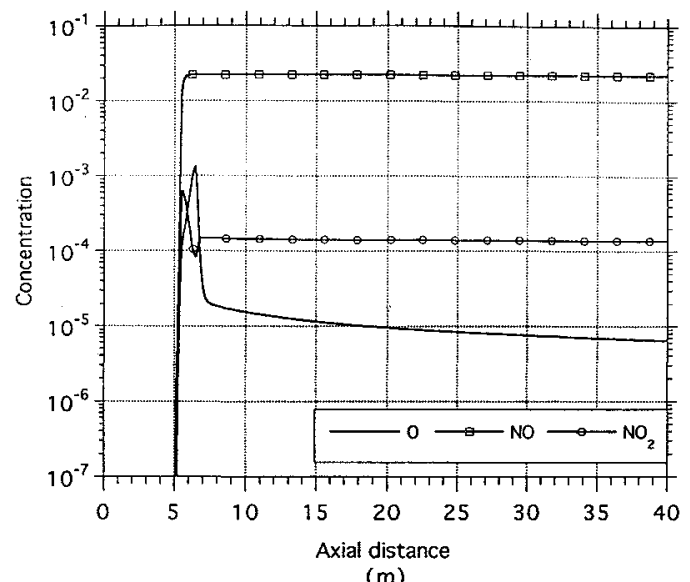

(m)

Fig. 10 Nonequilibrium concentrations for case 2.

a major engineering challenge. Other candidate laser sources for energy transfer include the nuclear reactor pumped Xe laser operating at $2.76 \mu$, the free electron laser with its variable frequency operation, the chemical oxygen iodine laser that runs at $1.4 \mu$, high efficiency diode lasers operating in the near infrared, the carbon dioxide laser in the $10-\mu$ region, and the carbon monoxide laser in the 5- $\mu$ region. In all cases, significant scale-ups will be required to generate enough power to drive a full-scale facility.

Other aspects that must be examined include optical propagation through the high-temperature air and the possible interaction of high-power laser beams with the walls. Of particular concern is electric-field-generated breakdown in the energy-addition region of the flow. Although this occurs at very high intensities for laser radiation, the variation of breakdown as a function of pressure is of concern. It is, perhaps, encouraging to note that the breakdown threshold reaches a minimum with pressure, which in air at the $\mathrm{CO}_{2}$ laser wavelength occurs at approximately 20 -atm pressure. At pressures higher than that, the breakdown threshold begins to increase since the breakdown dynamics become dominated by collisional processes. ${ }^{9}$ Breakdown is also very strongly dependent on the frequency of the electric field, with the breakdown threshold being much higher for high frequency, i.e., near infrared and visible lasers, than for low frequency, i.e., far infrared lasers or microwave devices. Another complexity that will have to be investigated is the interaction of the source radiation field with the two-dimensional flow structure including the boundary layer. The high-temperature boundary layer near the walls will cause the air density to be lower there than in the central portion of the flow. This density variation will lead to an index-of-refraction contour that may be used with the geometry shown in Fig. 4 to help guide the lasers and avoid excess wall heating.

Alternate methods for depositing energy downstream of the throat need to be examined. A particularly attractive approach is to use very high-power microwave gyrotrons to drive molecular oxygen in the vicinity of $60 \mathrm{GHz} .{ }^{10,11}$ The very rapid coupling of the oxygen molecule to the translational kinetic modes in air suggests that this approach can potentially be used to deposit large amounts of energy. Microwave gyrotron sources in the megawatt region are currently available, and an increase of power on the order of a factor of 10 can be anticipated in the near future, particularly given that continuous operation may not be required for this facility.

\section{Conclusions}

These preliminary examples suggest that a radiatively driven hypersonic air facility may be feasible. Major questions still remain, particularly regarding the effects of transport phenomena at high pressure, the energy-addition mechanism, and the associated chemical kinetics. The basic concept of achieving high enthalpy by using ultrahigh pressure and coupling energy in downstream of the throat does, in principle, lead to a wind-tunnel apparatus in which the air remains cool compared with other approaches. High-pressure plena have been constructed in Russia, and ultrahigh pressures have been achieved in small-scale laboratory devices. Issues relating to gas transport also must be further examined. These include such critical considerations as nozzle erosion, wall cooling, and nonequilibrium phenomena. Although these questions will require significant research to be done in a variety of fields, it is worthwhile to point out that the development of a true air hypersonic test facility will have a major impact on the design and testing of hypersonic vehicles.

\section{Acknowledgments}

This work was supported by the Air Force Arnold Engineering Development Center (AEDC) under the supervision of Keith Kushman. The concept was initially formulated in January of 1992 and significantly developed through the undergraduate research efforts of Jeffrey Guest in the spring of 1992 and Douglas Natelson the following year. Additional student support was provided through an Air Force Office of Scientific Research AASERT grant. A series of four workshops held at AEDC, Phillips Laboratory, NASA Langley, and Princeton University served to further establish the technical details and developmental challenges. Particular thanks are given to Marc Costantino of Lawrence Livermore Laboratories, who has contributed his expertise in high-pressure technologies along with his enthusiasm and encouragement. We also recognize the valuable suggestions of our Russian colleagues and greatly appreciate their willingness to share their high-pressure technologies with us.

\section{References}

${ }^{1}$ Mader, C. L., Numerical Modeling of Detonations, Univ. of California Press, Berkeley, CA, 1979, pp. 412-448.

${ }^{2}$ Reynolds, W. C., Thermodynamic Properties in SI, Dept. of Mechanical Engineering, Stanford Univ., Stanford, CA, 1979, pp. 157-159.

${ }^{3}$ Brahinsky, H. S., and Northcutt, D., Mollier Diagram for Equilibrium Air, von Kármán Gas Dynamics Facility, Arnold Engineering Development Center, Parthenon Press, Nashville, TN, 1967.

${ }^{4}$ Sychev, V. V., Vasserman, A. A., Kozlov, A. D., Spiridonov, G. A., and Tsymarny, V. A., Thermodynamic Properties of Air, Hemisphere, Washington, DC, 1987, pp. 119-199.

${ }^{5}$ Sychev, V. V., Vasserman, A. A., Kozlov, A. D., Spiridonov, G. A., and Tsymarny, V. A., Thermodynamic Properties of Nitrogen, Hemisphere, Washington, DC, 1987, pp. 143-236.

${ }^{6}$ Kiyama, R., "The Compressibility of the Air Under Ultra Pressure," Review of Physical Chemistry of Japan, Vol. 19, No. 1, 1945, pp. 38-42 (in Japanese).

${ }^{7}$ Rothman, L. W., Gamache, R. R., Goldman, A., Brown, L. R., Toth, R. A., Pickett, H. M., Poynter, R., Flaud, J.-M., Camy-Peyret, C., Barbe, A., Husson, N., Rinsland, C. P., and Smith, M. A. H., "The HITRAN Database: 1986 Edition," Applied Optics, Vol. 26, No. 19, 1987, pp. 4058-4097.

${ }^{8}$ Frederick, J. E., and Abrams, R. B., "Model Studies of Nitric Oxide Fluorescence in the Earth's Backscattered Spectrum," Planetary and Space Science, Vol. 30, No. 2, 1982, pp. 137-145.

${ }^{9}$ Raizer, Y. P., Gas Discharge Physics, Springer-Verlag, Berlin, 1991, p. 154 .

${ }^{10}$ Cassidy, P., Boeing Corporation, and Jory, H., Varian Corporation, private communications, 1993.

${ }^{11}$ Mingelgrin, U., Gordon, R. G., Frenkel, L., and Sullivan, T. E., "Microwave Spectrum of Compressed $\mathrm{O}_{2}$-Foreign Gas Mixtures in the 48-81 GHz Region," Journal of Chemical Physics, Vol. 57, No. 7, 1972, pp. 2923-2931. 